\title{
Abnormalities of diffusional kurtosis imaging and regional homogeneity in idiopathic generalized epilepsy with generalized tonic-clonic seizures
}

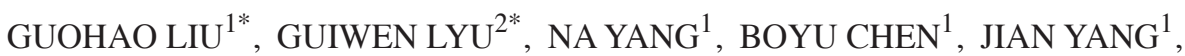 \\ YIWEN HU $^{1 *}$, YI LEI ${ }^{2}$, JUN XIA $^{2}$, FAN LIN $^{2}$ and GUOGUANG FAN ${ }^{1}$ \\ ${ }^{1}$ Department of Radiology, The First Affiliated Hospital of China Medical University, Shenyang, Liaoning 110001; \\ ${ }^{2}$ Department of Radiology, The Second People's Hospital of Shenzhen, Shenzhen, Guangdong 518035, P.R. China
}

Received April 12,2017; Accepted December 15, 2017

DOI: $10.3892 / \mathrm{etm} .2018 .7018$

\begin{abstract}
Neuroimaging techniques have been used to investigate idiopathic generalized epilepsy with generalized tonic-clonic seizures (IGE-GTCS) and different studies employing these methods have produced varying results. However, there have been few studies exploring diffusional kurtosis imaging (DKI) and regional homogeneity (ReHo) techniques in patients with IGE-GTCS. In the current study, resting-state functional magnetic resonance imaging (fMRI) and DKI data were collected from 28 patients with IGE-GTCS and 28 healthy controls. The ReHo method and tract-based spatial statistical (TBSS) analysis were performed to compare differences between the groups. Compared with healthy controls, patients with IGE-GTCS exhibited markedly increased ReHo in the bilateral putamen, the thalamus, right pallidum, right supplementary motor area and the bilateral paracentral lobules. Compared with healthy controls, patients with IGE-GTCS also exhibited markedly decreased $\mathrm{ReHo}$ in the posterior cingulate/precuneus, left angular gyrus and dorsolateral prefrontal cortex. In patients with IGE-GTCS, DKI revealed lower fractional anisotropy in the left anterior/superior corona radiata, left superior longitudinal fasciculus and genu/body of the corpus callosum. Higher mean diffusivity was detected in the bilateral anterior corona radiata, left superior corona radiata, left cingulum, and genu/body/splenium of the corpus callosum. Furthermore, reduced mean kurtosis values were identified over the bilateral
\end{abstract}

Correspondence to: Professor Guoguang Fan, Department of Radiology, The First Affiliated Hospital of China Medical University, 155 Nanjing North Street, Shenyang, Liaoning 110001, P.R. China

E-mail: fanguog1968@163.com

*Contributed equally

Key words: generalized tonic-clonic seizures, regional homogeneity, diffusional kurtosis imaging, tract-based spatial statistics superior/posterior corona radiate, left anterior corona radiata, right superior longitudinal fasciculus, left posterior thalamic radiation and the genu/body/splenium of the corpus callosum. Therefore, the results of the current study revealed abnormalities in spontaneous activity in the gray and white matter tracts in patients with IGE-GTCS. These results suggest that novel MRI technology may be useful to help determine the pathogenesis of IGE-GTCS.

\section{Introduction}

Idiopathic generalized epilepsy (IGE) is a type of epilepsy in which patients experience generalized tonic-clonic, myoclonic and absence seizures (1). Patients undergoing generalized tonic-clonic seizures (GTCS) are unresponsive as they sustain convulsions. Scalp electroencephalography (EEG) reveal that such patients exhibit generalized spike-and-wave discharges (GSWDs) of 2.5-5.0 Hz, which are thought be propagated, at least in part, via the corticothalamic circuit (2). Regional homogeneity (ReHo) is a voxel-based measure of brain activity, which evaluates the synchronization between different brain regions by calculating the similarity between the time series of a voxel and its proximal voxels. Requiring no prior knowledge about hemodynamic response, as a data-driven method, the major advantage is its ability to detect the changes of deoxyhemoglobin in venous blood. Abnormal regional homogeneity is relevant to the changes of temporal aspects of neural activity in the regional brain (3).

ReHo may be used clinically to evaluate functional modulations when the brain is in its resting state; many valuable findings have been obtained from ReHo conducted in patients with neuropsychological diseases, including epilepsy (4-6), schizophrenia (7) and amnestic mild cognitive impairment (8). The results of previous studies support that there is increased synchronization in the epileptogenic zones during seizures and the when patients are in the interictal state, which may be involved in the generation of interictal activity (5). It is also notable that ReHo analyses in patients with generalized epilepsy experiencing absence seizures (6) or patients with juvenile myoclonic epilepsy (4) have identified abnormalities in the striato-thalamo-cortical network and the thalamo-motor 
cortical network, respectively. In addition, convulsions experienced by patients with IGE may be caused by the hyperexcitability of motor neuron circuits. Thus, the thalamus, basal ganglia and motor cortex may all be involved in the development of IGE-GTCS. To evaluate changes in the spontaneous brain activity of patients with IGE-GTCS, the present study investigated the ReHo features of such patients compared with healthy controls. The effects of changes in ReHo on the clinical factors of IGE-GTCS were also assessed.

Although numerous studies have focused on the gray matter of patients with IGE-GTCS, few studies have assessed the white matter of these patients. Diffusion tensor imaging (DTI) studies have been used to determine structural abnormalities in patients with IGE-GTCS (9). However, it has been demonstrated that diffusional kurtosis imaging (DKI) is more sensitive than conventional DTI at evaluating tissue microstructure (10), even in the presence of crossing fibers. DKI is a novel magnetic resonance imaging (MRI)-based, noninvasive technique able to measure fractional anisotropy (FA), mean diffusivity (MD) and mean kurtosis (MK), and therefore may identify microstructural changes in the cerebral white matter (WM) (11). Tract-based spatial statistics (TBSS) represents a novel approach and provides a fully automated and independent analysis of multiple subjects, allowing for clear observation of localized changes in diffusion (12). Therefore, combining DKI with TBSS (DKI-TBSS) in patients with IGE-GTCS may allow the detection of alterations that were previously undetectable in the microstructure of the cerebral WM. Indeed, previous studies have identified changes in kurtosis-based diffusion metrics in several neuropathologies, including acute cerebral infarction (13), idiopathic normal pressure hydrocephalus (14) and epilepsy (15).

Most research into epilepsy has been conducted in patients with IGE or has used a single method to study a specific subtype of IGE. GTCS is the most common phenotype of IGE (16); therefore, the aim of the present study was to use DKI-TBSS to determine non-Gaussian diffusion patterns in the whole-brain WM of patients with IGE-GTCS. The characterization of such anatomical and functional connectivity abnormalities may improve the diagnosis and treatment of patients with early stage IGE-GTCS.

\section{Materials and methods}

Participants. A total of 28 right-handed patients (mean age, 22.85 \pm 5.61 years; 11 females and 17 males) with IGE-GTCS from the Epilepsy Clinic at the First Affiliated Hospital of China Medical University (Shenyang, China) were recruited between March 2016 and December 2016. IGE-GTCS was diagnosed based on the electroclinical criteria of the International League Against Epilepsy (17). The inclusion criteria for patients in the current study were as follows: i) The presence of typical clinical symptoms of IGE-GTCS, apart from partial seizures secondary to GTCS; ii) $\geq 1$ EEG examination identifying typical GSWDs on a normal background; iii) no abnormal or unusual clinical MRI findings; and iv) no typical GTCS seizures for 7 days. A total of 28 right-handed healthy participants (mean age, $24.52 \pm 4.34$ years; 12 females and 16 males) with no previous neurological or psychiatric problems were recruited as controls. There were no differences in the sex, age, education level and handedness between patients and controls (Table I). The present study was approved by the Ethics Committee of the First Hospital of China Medical University and written informed consent was obtained from all participants.

Image acquisition. Data were obtained on a SIEMENS Verio 3.0 Tesla MRI scanner (Siemens Healthineers, Erlangen, Germany). T2-weighted axial/oblique coronal images and fluid-attenuated inversion recovery oblique coronal images were acquired as part of the examination. All images underwent visual analysis by experienced radiologists and were found to be normal. Resting-state functional data were obtained using an echo-planar imaging sequence [repetition time (TR), 2,000 msec; echo time (TE), $30 \mathrm{msec}$. The following imaging parameters were used: Field of view, $240 \times 240 \mathrm{~mm}^{2}$; slice thickness, $4 \mathrm{~mm}$ with a $0.8 \mathrm{~mm}$ gap; and matrix, 64x64. DKI was applied to a single shot echo-planar imaging sequence (TR, 9,500 msec; TE, $104 \mathrm{msec}$ ) with the following diffusion weightings applied along 30 non-collinear directions: $b$-value, $0,1,000$ and $2,000 \mathrm{sec} / \mathrm{mm}^{2}$. A total of 45 axial slices were collected from each participant. The field of view was $222 \times 222 \mathrm{~mm}^{2}$ and slice thickness was $2 \mathrm{~mm}$ with no gap. Three-dimensional T1-weighted images using a magnetization-prepared rapid gradient echo sequence (TR, 2,250 msec; TE, $4.18 \mathrm{msec}$ ) were obtained for each participant. Imaging parameters were as follows: $256 \times 256 \mathrm{~mm}^{2}$ field of view, 1-mm slice thickness and 256x256 acquisition matrix.

ReHo analysis. All resting-state functional MRI (fMRI) data were pre-processed using the Data Processing Assistant for Resting-State fMRI (DPARSF_V2.2; restfmri. net/forum/dparsf_v2_2), which was based on Statistical Parametric Mapping (SPM; http://www.fil.ion.ucl.ac.uk/spm). To ensure magnetic field stabilization, the first 10 points were discarded. The remaining 230 points were corrected for slice timing and head motion, and then spatially normalized. T1-weighted images were spatially normalized based on the Montreal Neurological Institute (MNI) standard brain space (18), followed by detrending and bandpass filtering (0.01-0.08 Hz).Using Kendall'scoefficient of concordance, ReHo was computed using REST software (REST plus version 1.2; www.restfmri.net/forum/REST). ReHo was used to measure local synchronization of blood-oxygen-level-dependent fluctuations within 27 voxels in a voxel-wise manner. All ReHo maps were smoothed with a Gaussian kernel of $6 \times 6 \times 6 \mathrm{~mm}^{3}$ (full-width at half-maximum). To evaluate local spontaneous brain activity in each group of participants, a one-sample $t$ test was used to compare ReHo values. Two-sample t-tests based on ReHo maps were used to evaluate differences between patients with IGE-GTCS and healthy controls. P $<0.05$ was considered to indicate a significant difference. False discovery rate correction was used for multiple comparisons as well as cluster correction for a cluster size $\geq 21$ voxels. The REST Toolbox was used to obtain cluster sizes, locations and their respective t-values. The $t$-value represented the statistical value of the peak voxel that exhibited differences in ReHo and compared these values between patients with IGE-GTCS and healthy controls. Finally, the correlation between ReHo 
Table I. Demographic and clinical characteristics of patients with IGE-GTCS and HCs.

\begin{tabular}{lccc}
\hline Clinical characteristics & Patients with IGE-GTCS (n=28) & Patients with HC (n=28) & P-values \\
\hline Age (years) & $22.85 \pm 5.61$ & $24.52 \pm 4.34$ & 0.253 \\
Sex (female:male) & $11: 17$ & $12: 16$ & 0.766 \\
Duration of epilepsy (years) & $3.47 \pm 1.68$ & $\mathrm{n} / \mathrm{a}$ & $\mathrm{n} / \mathrm{a}$ \\
Duration of education (years) & $11.06 \pm 1.96$ & $11.89 \pm 2.12$ & 0.570 \\
Handedness (right/left) & $28 / 0$ & $28 / 0$ & $>0.99$
\end{tabular}

Data are expressed as the range from min-max or as the mean \pm standard deviation. IGE-GTCS, idiopathic generalized epilepsy with generalized tonic-clonic seizures; HC, healthy controls.

values and epilepsy duration were determined using Pearson's correlation for patients with IGE-GTCS.

DKI image processing with TBSS. All diffusion images without lesions, artifacts or severe atrophy for brain distortion were corrected using the 'eddy-current' toolbox in the Functional MRI of the Brain (FMRIB) Software Library (FSL) tools (version 5.0; www.fmrib.ox.ac.uk/fsl) for Linux. Subsequently, the Diffusion Kurtosis Estimator (DKE) (www.nitrc.org/projects/dke) was used to calculate DKI parametric maps, including MK, FA and MD. DKI data were normalized to the Montreal Neurological Institute (MNI) standard brain space (18) using the FMRIB Software Library (FSL) tools. FA maps of all participants were aligned to the MNI152 space. Subsequently, the mean FA image was generated and thinned to create the mean FA skeleton, which represented the centers of all tracts common to each group. An FA threshold of 0.2 was applied to exclude further prevent partial-volume effects, and peripheral tracts and gray matter were excluded $(15,19)$. A threshold was applied to the mean FA skeleton and a skeletonized FA map was made by projecting each participant's FA map onto the mean FA skeleton with local maximum FA values. This projection was also applied to other non-FA diffusion metrics to create skeletonized MD and MK maps. Permutation-based testing was used with 5,000 permutations and statistical inferences by threshold-free cluster enhancement, with a threshold of $\mathrm{P}<0.05$, corrected for multiple comparisons (family wise error) using random field theory. Significant differences in the anatomic locations of white matter tracts were revealed by TBSS. The JHU ICBM-DTI-81 White-Matter Labels Atlas (20) was used to evaluate results. The values of MK, FA, and MD were calculated for each cluster using in-house Matlab scripts (Matlab 2012b, Math Works, Inc., Natick, MA, USA). Student's t test was used to compare the values of MK, FA and MD in the area of the WM between IGE-GTCS patients and healthy controls. The t-value represented the statistical value of peak voxel identifying differences in MK, FA and MD differences between patients with IGE-GTCS and healthy controls. FA, MD and MK values from each participant were extracted from regions exhibiting significant differences between patients and controls in TBSS. Partial Spearman's correlation analysis was performed to evaluate the association between extracted values and epilepsy duration following controlling for age and sex.

\section{Results}

Within-group and between-group ReHo analyses. Differences in ReHo values within each group were analyzed using a one-sample t-test. Compared with healthy controls, ReHo values were markedly increased in patients with IGE-GTCS in bilateral regions of the basal-thalamus, including the putamen, thalamus, right pallidum, right supplementary motor area and bilateral paracentral lobules (Fig. 1 and Table II). However, ReHo were values were markedly decreased in the posterior cingulate cortex, left angular gyrus, left middle frontal gyrus and left superior fontal gyrus (Fig. 2 and Table II). ReHo and epilepsy duration were negatively correlated in regions of the right superior frontal gyrus ( $\mathrm{r}=-0.693, \mathrm{P}=0.003$; Fig. 3).

DKI-TBSS. Compared with healthy controls, patients with IGE-GTCS exhibited microstructural abnormalities in their WM. A large cluster of significantly reduced FA was detected in patients with IGE-GTCS compared with controls (Table III). Affected WM tracts included the left anterior corona radiata, left superior corona radiata, left superior longitudinal fasciculus and genu/body of the corpus callosum (MNI coordinates of local maxima, -8/7/26). Two large clusters of significantly increased MD were observed in the WM tracts of patients with IGE-GTCS compared with healthy controls (Table IV). Affected WM tracts included the bilateral anterior corona radiata, left superior corona radiata, left cingulum, genu/body of the corpus callosum (MNI coordinates of local maxima, $5 / 27 / 0$ ); and left splenium/body of the corpus callosum (MNI coordinates of local maxima, -12/-41/11). Four large clusters of significantly decreased MK values were observed in the WM tracts of patients with IGE-GTCS compared with controls (Table V). Affected WM tracts included the right superior longitudinal fasciculus (MNI coordinates of local maxima, 41/-37/32); left posterior thalamic radiation (including optic radiation; MNI coordinates of local maxima=-28/-65/17); bilateral superior corona radiata, bilateral posterior corona radiata, left anterior corona radiata, left cingulum and splenium/genu/body of the corpus callosum (MNI coordinates of local maxima, -12/-41/21); and left superior corona radiata (MNI coordinates of local maxima, -28/-14/23). Notably, the abnormalities that manifested as decreased $\mathrm{MK}$ values were more extensive. However, the abnormalities revealed by kurtosis metrics did not fully explain the abnormalities that manifested as reduced FA and increased MD (Fig. 4). 
A

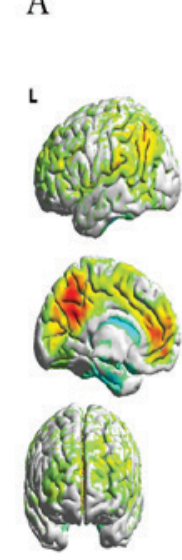

$\mathrm{HC}$

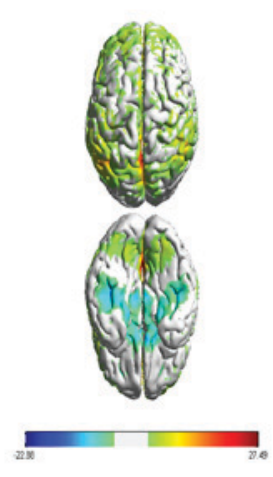

B

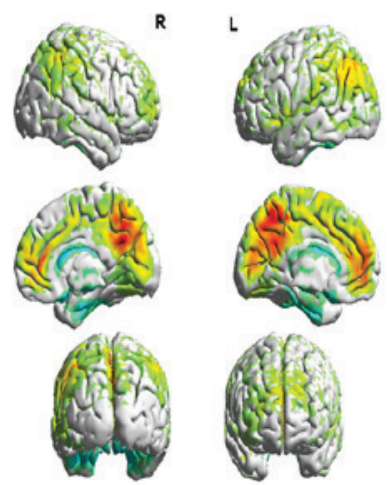

IGE-GTCS

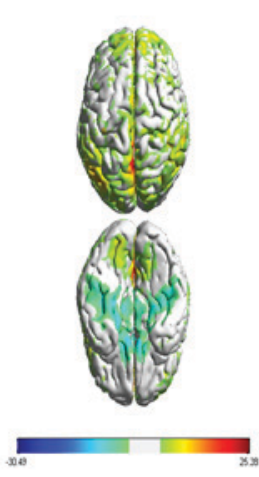

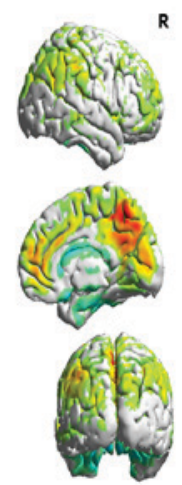

Figure 1. ReHo results from (A) healthy controls and (B) patients with IGE-GTCS of a one-sample t-test. Red-orange indicates an increase in ReHo whilst blue-green indicates a decrease in ReHo. ReHo, regional homogeneity; L, left; R, right.

There was no correlation observed between DKI metrics and epilepsy duration (Table VI).

\section{Discussion}

Amplitude of low-frequency fluctuation (21), functional connectivity (22) and ReHo are resting-state fMRI methods that may be used to assess patients with IGE-GTCS. ReHo reflects the synchronization of neural activity in local brain regions (3). In the present study, analysis of within- and between-group ReHo measurements identified widespread cortical and subcortical region involvement. Compared with healthy controls, patients with IGE-GTCS presented with significantly increased ReHo values in bilateral basal ganglia-thalamus regions and regions of the cortex associated with motor function, including the supplementary motor area and bilateral paracentral lobules. The thalamus, which regulates susceptibility to and propagation of seizures, is the major relay station of the cortical and subcortical projection systems. The thalamus may serve as a stronger driver of cortical activity, from initiation to propagation of GSWDs (23) and may be critical for the generation of epileptic tonic-clonic motor activity and impairment of consciousness (24). Paz et al (25) used optogenetics in a rat model to reveal the crucial role of the thalamus within the cortico-thalamo-cortical network in sustaining seizures. Furthermore, structural investigations centered on voxel-based morphometry demonstrated that the volume of gray matter is reduced in the thalami of patients with IGE-GTCS (26).

Basal ganglia are widely considered to mediate epileptic discharge regulation (27) and their dysfunction is associated with locomotor disturbances, which may be linked to motor responses as uncontrolled jerking movements in tonic-clonic seizures. It has been reported that patients with idiopathic generalized epilepsy syndromes exhibit reduced putamen volumes (28). Furthermore, reduced dopamine transporter binding has been detected in the putamen of patients with IGE-GTCS, who primarily exhibited impairments in motor control and speed, which suggests that dopamine may be neuroprotective and may inhibit the onset of seizures (29). In addition, the substantia nigra may mediate the spread of epileptic activity (30). The imaging threshold set by
SPM may result in brain areas appearing smaller than they actually are and may account for the absence in the results of the current study regarding small basal ganglia nuclei. Somatotopic organization of movement-related neurons is maintained throughout the basal ganglia-thalamo-cortical circuit (31). Additionally, functional connectivity between the putamen and the motor and premotor cortices has been previously reported (32). Furthermore, the pallidum and putamen comprise a resting state network in the basal ganglia, which also project into the supplementary motor area, functioning as the motor control circuit of the basal ganglia (31). Most patients with IGE-GTCS present with sustained muscle rigidity and rapid muscle contractions during seizures; therefore, the current study hypothesized that there would be disruptions in the basal ganglia-thalamo-cortical circuit. It is likely that the basal ganglia themselves do not generate seizures; however, cortical feedback loops mediated by basal ganglia circuits may impact on cortical epileptic activity (33).

In the present study, ReHo values were decreased in the posterior cingulate cortex (PCC) and left angular gyrus. These areas overlap with the default mode network (DMN), which influences self-awareness, episodic memory and interactive modulation (34). In the current study, decreased activity was observed in the dorsolateral prefrontal cortex (DLPFC), which is the central brain region of the central executive network (CEN) that mediates cognitive and emotional circuits (35). Wei et al (36) used Granger causality analysis to identify alterations in direct causal relationships across the key nodes PCC and DLPFC, of the DMN and CEN, respectively, in patients with IGE-GTCS. Relative to the healthy control group, patients with IGE-GTCS demonstrated a significantly enhanced Granger causal influence from the DLPFC to the PCC, which is coherent in both time and frequency domain analysis, and the results were consistent with the results of the current study. The altered efficacy in connectivity between the DLPFC and the dorsal anterior cingulate cortex may be a key factor that affects cognitive dysfunction in patients with IGE-GTCS (36). It has also been demonstrated that PCC is primarily involved in determining consciousness. Laureys et al (37) identified that the PCC serves a pivotal role in regulating consciousness due to its anatomic location, as it links to the anterior thalamic 
Table II. Brain regions of increased/decreased ReHo in patients with IGE-GTCS.

\begin{tabular}{|c|c|c|c|c|c|c|c|c|}
\hline \multirow[b]{2}{*}{ Brain region } & \multirow[b]{2}{*}{ Voxels } & \multirow[b]{2}{*}{ AAL } & \multirow[b]{2}{*}{ BA } & \multirow[b]{2}{*}{ Side } & \multicolumn{3}{|c|}{ MNI coordinates } & \multirow[b]{2}{*}{ t-value } \\
\hline & & & & & $\mathrm{X}$ & $\mathrm{Y}$ & $\mathrm{Z}$ & \\
\hline Putamen & 95 & 73 & & $\mathrm{~L}$ & -29 & -4 & -3 & 3.029 \\
\hline Putamen & 71 & 74 & & $\mathrm{R}$ & 24 & -1 & 8 & 2.522 \\
\hline Pallidum & 42 & 76 & & $\mathrm{R}$ & 21 & -5 & 8 & 2.003 \\
\hline Thalamus & 69 & 77 & & $\mathrm{~L}$ & -7 & -17 & 8 & 2.753 \\
\hline Thalamus & 32 & 78 & & $\mathrm{R}$ & 6 & -12 & 8 & 2.221 \\
\hline Supplementary motor area & 55 & 20 & 6 & $\mathrm{R}$ & 3 & -21 & 63 & 4.431 \\
\hline Paracentral lobule & 92 & 69 & & $\mathrm{~L}$ & -5 & -32 & 70 & 2.327 \\
\hline Paracentral lobule & 73 & 70 & & $\mathrm{R}$ & 5 & -32 & 69 & 2.510 \\
\hline Posterior cingulate cortex & 33 & 35 & 23 & $\mathrm{~L}$ & -1 & -36 & 30 & -2.132 \\
\hline Posterior cingulate cortex & 31 & 36 & 23 & $\mathrm{R}$ & 5 & -36 & 31 & -2.450 \\
\hline Angular gyrus & 151 & 65 & 39 & $\mathrm{~L}$ & -45 & -68 & 35 & -3.7735 \\
\hline Middle frontal gyrus & 116 & 7 & 46 & $\mathrm{~L}$ & -41 & 54 & 9 & -2.946 \\
\hline Superior frontal gyrus & 43 & 3 & 10 & $\mathrm{~L}$ & -27 & 63 & 17 & -4.311 \\
\hline
\end{tabular}

The t-value represents the statistical value of peak voxel exhibiting differences in ReHo in patients with IGE-GTCS compared with healthy control patients. Positive t-values indicate increased ReHo and negative t-values indicate decreased ReHo. ReHo, regional homogeneity; IGE-GTCS, idiopathic generalized epilepsy with generalized tonic-clonic seizures; AAL, Anatomical Automatic Labeling; BA, Brodmann's area; MNI, Montreal Neurologic Institute; L, left; R, right.

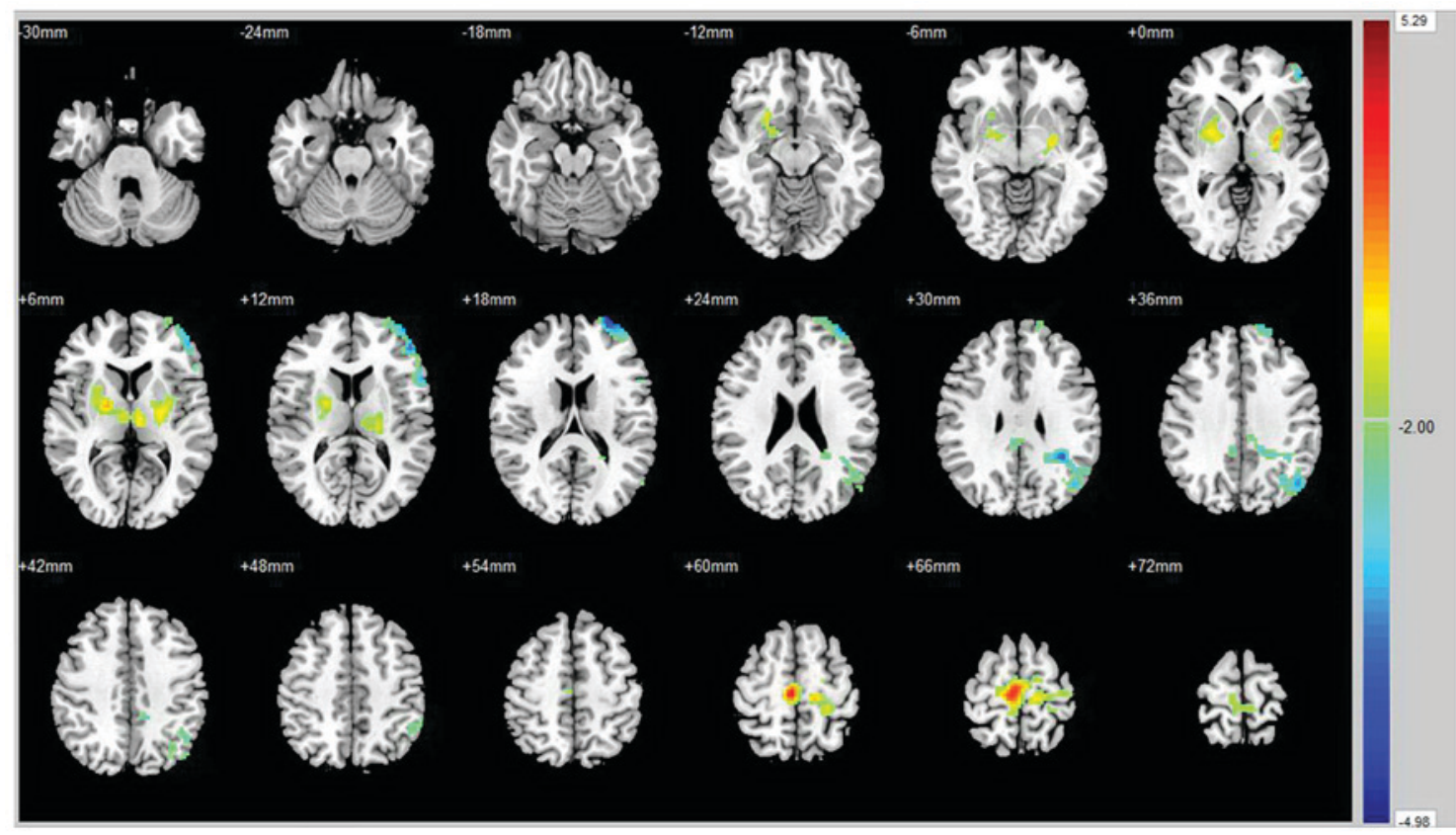

Figure 2. Brain maps indicating differences in ReHo between healthy controls and patients with idiopathic generalized epilepsy with generalized tonic-clonic seizures. Red and yellow indicate increased ReHo and blue indicates decreased ReHo. $\mathrm{P}<0.05$ was determined to indicate a significant difference and cluster size was $>21$ voxels, which corresponded to a corrected $\mathrm{P}<0.05$. The numbers in the upper left corners indicate $\mathrm{z}$-plane coordinates of the MNI space. The right hemisphere is represented on the left side of each image. MNI, Montreal Neurological Institute; ReHo, Regional homogeneity; MK, mean kurtosis; MD, mean diffusivity; FA, fractional anisotropy.

nucleus and thalamic arousal system of the brainstem. In the present study, a negative correlation was identified between ReHo in the right superior frontal gyrus regions and epilepsy duration. This suggests that patients with IGE-GTCS exhibit an increased vulnerability to seizure in the right superior frontal gyri.
In the present study, the DKI metrics MK, FA, and MD served as quantitative measures of microstructural changes in the brains of patients with IGE-GTCS. FA quantifies preferred direction and MD presents the average extent of water diffusion in WM. MK values are quantitative measures of non-Gaussian water diffusion, which results from barriers to 
Table III. MNI coordinates of the brain with reduced FA identified by TBSS.

\begin{tabular}{|c|c|c|c|c|c|c|c|c|}
\hline \multirow{2}{*}{$\begin{array}{l}\text { Cluster } \\
\text { number }\end{array}$} & \multirow[b]{2}{*}{ Voxels } & \multirow[b]{2}{*}{ Atlas } & \multirow[b]{2}{*}{ Side } & \multirow[b]{2}{*}{ P-values } & \multicolumn{3}{|c|}{ MNI coordinates } & \multirow[b]{2}{*}{$\mathrm{t}$-value } \\
\hline & & & & & $\mathrm{X}$ & $\mathrm{Y}$ & $\mathrm{Z}$ & \\
\hline \multirow[t]{5}{*}{1} & 4,027 & Body of corpus callosum & & 0.01 & -8 & 7 & 26 & 2.06 \\
\hline & 1,170 & Anterior corona radiata & $\mathrm{L}$ & 0.01 & & & & \\
\hline & 623 & Genu of corpus callosum & & 0.01 & & & & \\
\hline & 310 & Superior corona radiata & $\mathrm{L}$ & 0.01 & & & & \\
\hline & 107 & Superior longitudinal fasciculus & $\mathrm{L}$ & 0.01 & & & & \\
\hline
\end{tabular}

The t-value represents the statistical value of peak voxel exhibiting differences in FA in patients with IGE-GTCS compared with healthy control patients. IGE-GTCS, idiopathic generalized epilepsy with generalized tonic-clonic seizures; TBSS, tract-based spatial statistics; FA, fractional anisotropy; MNI, Montreal Neurologic Institute; L, left; R, right.
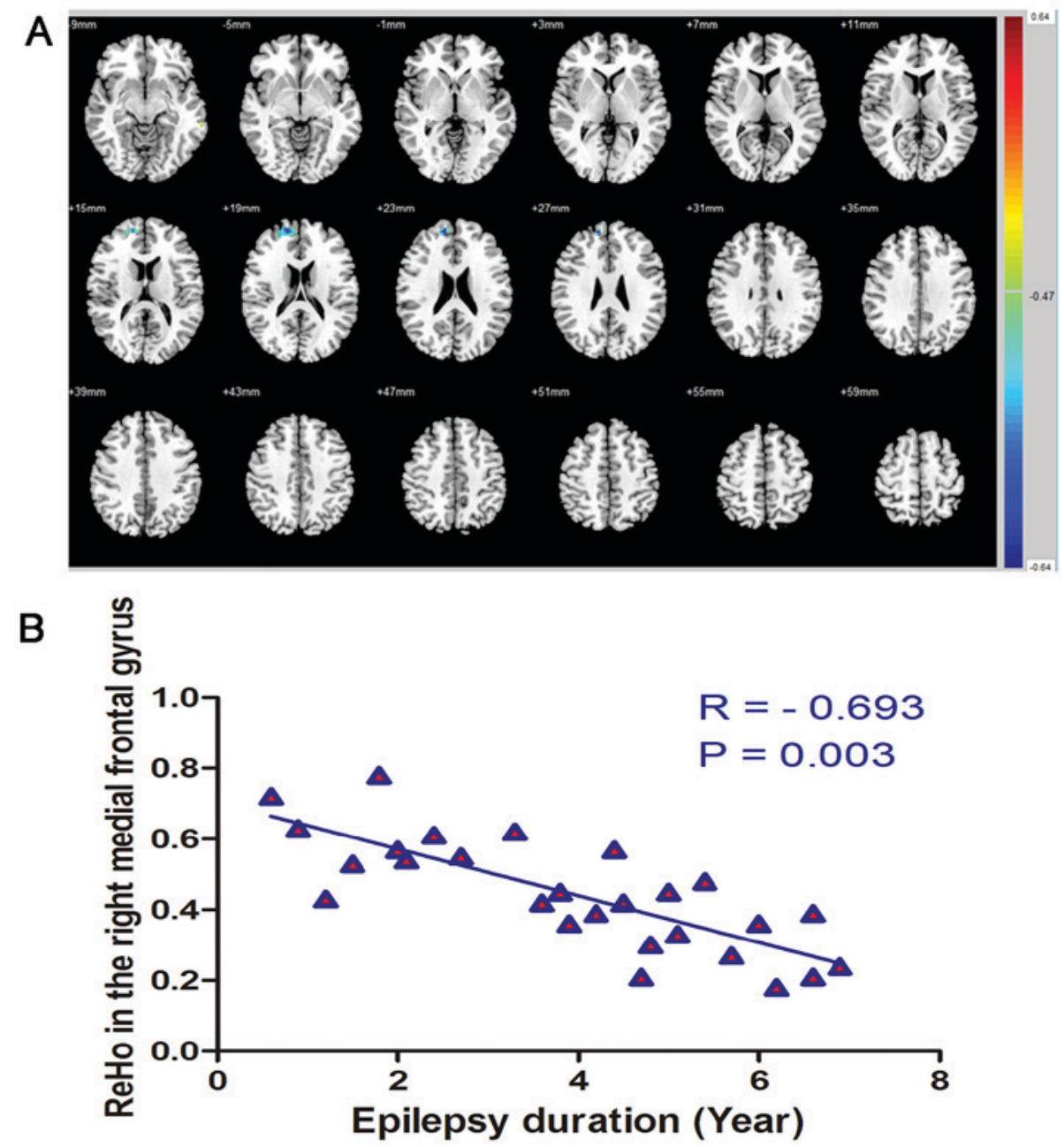

Figure 3. Correlation between epilepsy duration and ReHo in patients with idiopathic generalized epilepsy with generalized tonic-clonic seizures. (A) The peak voxel of ReHo in the right superior frontal gyrus $(x=17, y=57$ and $z=22)$ was measured. The right hemisphere is represented on the left side of each image. Blue indicates decreases in ReHo. (B) Peak ReHo in the right superior frontal gyrus was negatively correlated with epilepsy duration ( $\mathrm{r}=-0.683$; $\mathrm{P}=0.003)$. ReHo, Regional homogeneity.

diffusion (13). The pathophysiological mechanisms involved in determining the changes in FA and MD in patients with IGE-GTCS remain unknown. Decreased FA in WM is indicative of disrupted microstructural integrity; FA is affected by cell membrane and myelin integrity, as well as fiber density.
Increased MD is similarly indicative of disruption in microscopic barriers and accumulation of extracellular fluid, and has been observed in neuropathological diseases associated with tissue degeneration and edema (38). It is noteworthy that changes in FA and MD were primarily confined to the 
Table IV. MNI coordinates of the brain with increased MD identified by TBSS.

\begin{tabular}{|c|c|c|c|c|c|c|c|c|}
\hline \multirow{2}{*}{$\begin{array}{l}\text { Cluster } \\
\text { number }\end{array}$} & \multirow[b]{2}{*}{ Voxels } & \multirow[b]{2}{*}{ Atlas } & \multirow[b]{2}{*}{ Side } & \multirow[b]{2}{*}{ P-values } & \multicolumn{3}{|c|}{ MNI coordinates } & \multirow[b]{2}{*}{ t-value } \\
\hline & & & & & $\mathrm{X}$ & $\mathrm{Y}$ & $\mathrm{Z}$ & \\
\hline \multirow[t]{6}{*}{1} & 2,986 & Genu of corpus callosum & & 0.01 & 5 & 27 & 0 & 2.50 \\
\hline & 4,476 & Body of corpus callosum & & 0.01 & & & & \\
\hline & 541 & Anterior corona radiata & $\mathrm{R}$ & 0.01 & & & & \\
\hline & 327 & Anterior corona radiata & $\mathrm{L}$ & 0.01 & & & & \\
\hline & 176 & Superior corona radiata & $\mathrm{L}$ & 0.01 & & & & \\
\hline & 110 & Cingulum & $\mathrm{L}$ & 0.01 & & & & \\
\hline \multirow[t]{2}{*}{2} & 3,585 & Splenium of corpus callosum & $\mathrm{L}$ & 0.01 & -12 & -41 & 11 & 3.06 \\
\hline & 131 & Body of corpus callosum & $\mathrm{L}$ & 0.01 & & & & \\
\hline
\end{tabular}

The t-value represents the statistical value of peak voxel exhibiting differences in MD in patients with IGE-GTCS compared with healthy control patients. IGE-GTCS, idiopathic generalized epilepsy with generalized tonic-clonic seizures; TBSS, tract-based spatial statistics; MD, mean diffusivity; MNI, Montreal Neurologic Institute; L, left; R, right.

Table V. MNI coordinates of the brain with reduced MK identified by TBSS.

\begin{tabular}{|c|c|c|c|c|c|c|c|c|}
\hline \multirow{2}{*}{$\begin{array}{l}\text { Cluster } \\
\text { number }\end{array}$} & \multirow[b]{2}{*}{ Voxels } & \multirow[b]{2}{*}{ Atlas } & \multirow[b]{2}{*}{ Side } & \multirow[b]{2}{*}{ P-values } & \multicolumn{3}{|c|}{ MNI coordinates } & \multirow[b]{2}{*}{ t-value } \\
\hline & & & & & $\mathrm{X}$ & $\mathrm{Y}$ & $\mathrm{Z}$ & \\
\hline \multirow[t]{9}{*}{1} & 4,861 & Splenium of corpus callosum & & 0.01 & -12 & -41 & 21 & 2.97 \\
\hline & 3,927 & Body of corpus callosum & & 0.01 & & & & \\
\hline & 572 & Superior corona radiata & $\mathrm{L}$ & 0.01 & & & & \\
\hline & 328 & Posterior corona radiata & $\mathrm{L}$ & 0.01 & & & & \\
\hline & 319 & Cingulum & $\mathrm{L}$ & 0.01 & & & & \\
\hline & 152 & Superior corona radiata & $\mathrm{R}$ & 0.01 & & & & \\
\hline & 107 & Genu of corpus callosum & & 0.01 & & & & \\
\hline & 104 & Anterior corona radiata & $\mathrm{L}$ & 0.01 & & & & \\
\hline & 100 & Posterior corona radiata & $\mathrm{R}$ & 0.01 & & & & \\
\hline 2 & 390 & Superior longitudinal fasciculus & $\mathrm{R}$ & 0.01 & 41 & -37 & 32 & 2.60 \\
\hline 3 & 248 & Superior corona radiata & $\mathrm{L}$ & 0.01 & -28 & -14 & 23 & 2.73 \\
\hline 4 & 225 & Posterior thalamic radiation & $\mathrm{L}$ & 0.01 & -28 & -65 & 17 & 4.73 \\
\hline
\end{tabular}

The t-value represents the statistical value of peak voxel exhibiting differences in MK in patients with IGE-GTCS compared with healthy control patients. IGE-GTCS, idiopathic generalized epilepsy with generalized tonic-clonic seizures; TBSS, tract-based spatial statistics; MK, mean kurtosis; MNI, Montreal Neurologic Institute; L, left; R, right.

bilateral anterior and left superior corona radiate, the corpus callosum, cingulum and superior longitudinal fasciculus in the current study. As projection fibers, the anterior and superior corona radiata reciprocally connect the cerebral cortex to the thalamus. Similarly, the genu and body of the corpus callosum comprise interhemispheric commissural fibers that interconnect the prefrontal, premotor and supplementary motor areas (39). A previous EEG study revealed that the corpus callosum facilitates the epileptogenic susceptibility of the hemispheres via bisynchronous and bisymmetrical epileptiform discharges (40). The results of clinical studies support this hypothesis: Corpus callosotomy reduces the frequency and severity of generalized seizures in patients with medically intractable IGE (41).
In the current study, brain regions with changes in MK values were distinct from regions with changes in MD and FA. Reduced MK values were not limited to the WM tracts of the corpus callosum or corona radiata, but also included the WM of the posterior thalamic radiation (including optic radiation) and the right superior longitudinal fasciculus (SLF). Lower MK values indicate reduced diffusion heterogeneity, and there is a weak correlation between MK and MD in the brains of humans (11). In particular, reduced MK values in the brains of patients with IGE-GTCS may be associated with reduced cell compartmentalization and increased membrane permeability (42). It may also be useful to assess MK to evaluate crossing fibers, while FA and MD in WM may be affected by the presence of crossing fibers. MK is able to identify areas 
Table VI. Association between epilepsy duration and DKI in patients with IGE-GTCS.

\begin{tabular}{|c|c|c|c|c|c|c|c|}
\hline \multirow{2}{*}{$\begin{array}{l}\text { Cluster } \\
\text { number }\end{array}$} & \multirow{2}{*}{$\begin{array}{l}\text { DKI metrics } \\
\left(\mu \mathrm{m}^{2} / \mathrm{ms}\right)\end{array}$} & \multicolumn{3}{|c|}{ MNI coordinates } & \multirow{2}{*}{$\begin{array}{l}\text { IGE-GTCS } \\
\quad(\mathrm{n}=28)\end{array}$} & \multirow{2}{*}{$\begin{array}{c}\text { Duration of epilepsy } \\
\text { (years) }\end{array}$} & \multirow[b]{2}{*}{ P-values } \\
\hline & & $X$ & $\mathrm{Y}$ & $\mathrm{Z}$ & & & \\
\hline & FA & & & & & & \\
\hline \multirow[t]{2}{*}{1} & & -8 & 7 & 26 & $0.45 \pm 0.03$ & $3.47 \pm 1.68$ & 0.87 \\
\hline & MD & & & & & & \\
\hline 1 & & 5 & 27 & 0 & $0.94 \pm 0.03$ & $3.47 \pm 1.68$ & 0.38 \\
\hline \multirow[t]{2}{*}{2} & & -12 & -41 & 11 & $0.90 \pm 0.04$ & $3.47 \pm 1.68$ & 0.19 \\
\hline & MK & & & & & & \\
\hline 1 & & -12 & -41 & 21 & $0.94 \pm 0.04$ & $3.47 \pm 1.68$ & 0.56 \\
\hline 2 & & 41 & -37 & 32 & $0.88 \pm 0.05$ & $3.47 \pm 1.68$ & 0.63 \\
\hline 3 & & -28 & -14 & 23 & $0.95 \pm 0.04$ & $3.47 \pm 1.68$ & 0.50 \\
\hline 4 & & -28 & -65 & 17 & $0.98 \pm 0.04$ & $3.47 \pm 1.68$ & 0.71 \\
\hline
\end{tabular}

The diffusion parameters (FA, MD and MK) measured from spatially normalized skeleton DKI maps. Partial Spearman's correlation analysis was performed to examine the correlation between extracted values and epilepsy duration. Data are expressed as the mean \pm standard deviation. DKI, diffusional kurtosis imaging; IGE-GTCS, idiopathic generalized epilepsy with generalized tonic-clonic seizures; FA, fractional anisotropy; MD, mean diffusivity; MK, mean kurtosis; MNI, Montreal Neurologic Institute.

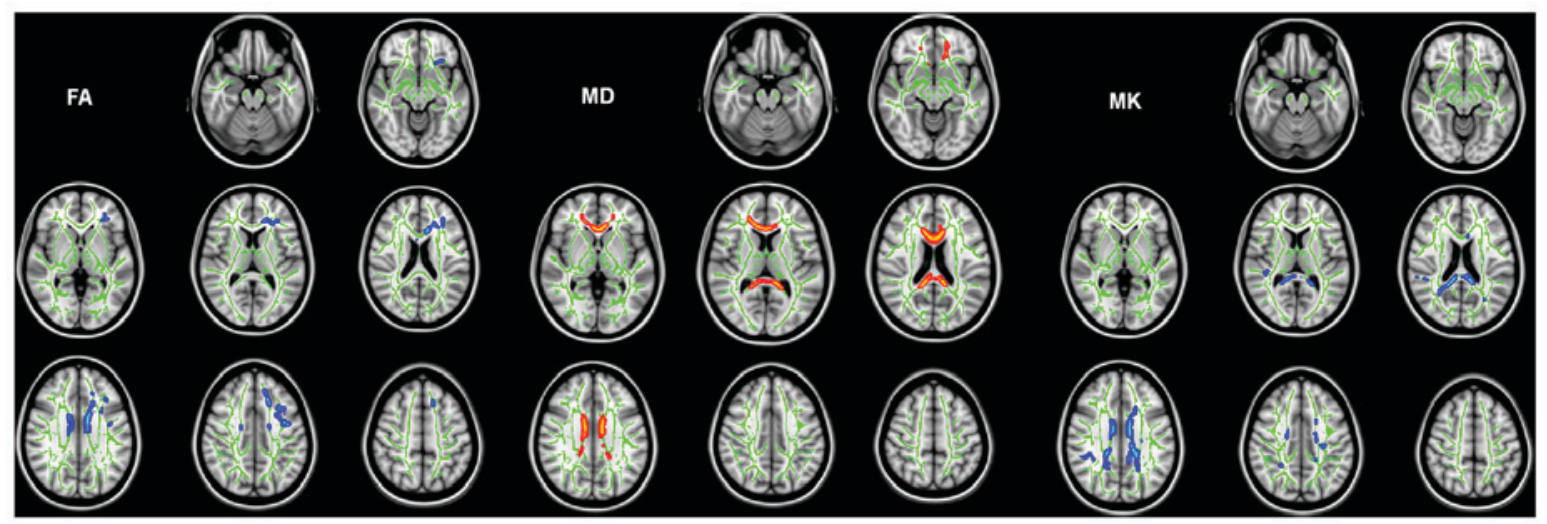

Figure 4. TBSS analysis of FA, MD and MK. The mean FA skeleton of all subjects (Green) was overlaid onto the MNI152 image. Regions with significant FA and MK reductions (highlighted in blue) and MD increases (highlighted in red-yellow) were superimposed onto the mean FA image and white matter skeleton (green) in patients with idiopathic generalized epilepsy with generalized tonic-clonic seizures compared with healthy controls. The right hemisphere is represented on the left side of each image. FA, fractional anisotropy; MD, mean diffusivity; MK, mean kurtosis; MNI, Montreal Neurological Institute; TBSS, tract-based spatial statistics.

of crossing fibers and this may explain the sensitivity of DKI to changes in cerebral WM (43). Reduced MK values in the posterior thalamic radiation (including optic radiation) may explain the visual aura experienced by some patients with IGE (44). SLF is involved in executive (inhibitory control) and language function (45). IGE-GTCS is traditionally considered to be a genetic form of epilepsy (17). However, no correlation was identified between DKI metrics and epilepsy duration in patients with IGE-GTCS. The results of the current study demonstrate that microstructural abnormalities may represent subtle neurodevelopmental alterations that precede the onset of epilepsy in IGE-GTCS (15). In general, the functional connectivity network is thought to be more flexible whilst the structural connectivity network is relatively stable. Therefore, the structural connectivity network may be affected less in patients with IGE-GTCS (16).
An unexpected result of the current study was that spatial distributions of FA were asymmetric in patients with IGE-GTCS. It is generally considered that changes in the brain are bilateral and symmetrical in IGE-GTCS; however, the results of the current study indicated that the left hemisphere was more affected than the right. These results are consistent with those of a previous study (46) that indicated that patients with IGE-GTCS exhibit brain asymmetry and lateralization of features, the tendency for some neural functions or cognitive processes to be specialized to one side of the brain or the other. Forced ictal head version or asymmetric tonic limb posturing are highly informative regarding seizure lateralization (47). The cause of seizure lateralization in IGE-GTCS is not well understood. Epileptic activity affecting the two hemispheres unequally may lead to lateralization of features that can be observed in IGE-GTCS (47). 
In conclusion, the current study investigated the pattern of regional hemodynamic synchronization in the brains of patients with IGE-GTCS by performing ReHo analysis of resting-state fMRI scans. Patients with IGE-GTCS exhibited altered regional synchronization in the bilateral thalami, the basal ganglia, motor-related cortex, posterior DMN regions and the CEN regions. Different DKI indices indicated different sensitivities for the detection of changes in diffusion. Regions exhibiting MK alterations were distinct from the regions with MD and FA abnormalities, suggesting that the role of DKI-TBSS in the characterization of microstructural characteristics of the brains of patients with IGE-GTCS differs from that of conventional DTI. These results, which support the potential of ReHo and DKI-TBSS as techniques for detecting intrinsic epileptic activity, provide important insights into the understanding of the pathophysiological mechanisms involved in IGE-GTCS.

\section{Acknowledgements}

Not applicable.

\section{Funding}

The present study was supported by the Science and Technology Development Program of Shenzhen (grant no. JCYJ20150731154850923).

\section{Availability of data and materials}

All datasets used and/or generated during the current study are available from the corresponding author on reasonable request.

\section{Authors' contributions}

GF, GLi and GLy designed the study. GLi and NY collected the data. GLi, GLy, BC, JY, YH, YL, JX and FL analyzed the data. GF and GLi prepared the manuscript. All authors read and approved the final manuscript.

\section{Ethics approval and consent to participate}

The current study was approved by the Ethics Committee of the First Affiliated Hospital of China Medical University (Shenyang, China). All patients provided written informed consent.

\section{Patient consent for publication}

All participants provided written informed consent for publication of their data.

\section{Competing interests}

The authors declare that they have no competing interests.

\section{References}

1. Ferrie CD: Idiopathic generalized epilepsies imitating focal epilepsies. Epilepsia 46 (Suppl 9): S91-S95, 2005.
2. Szaflarski JP, Kay B, Gotman J, Privitera MD and Holland SK: The relationship between the localization of the generalized spike and wave discharge generators and the response to valproate. Epilepsia 54: 471-480, 2013.

3. Zang Y, Jiang T, Lu Y, He Y and Tian L: Regional homogeneity approach to fMRI data analysis. NeuroImage 22: 394-400, 2004.

4. Jiang S, Luo C, Liu Z, Hou C, Wang P, Dong L, Zhong C, Lai Y, $\mathrm{Xia} Y$ and Yao D: Altered local spontaneous brain activity in juvenile myoclonic epilepsy: A preliminary resting-state fMRI study. Neural Plast 2016: 3547203, 2016.

5. Zeng H, Pizarro R, Nair VA, La C and Prabhakaran V: Alterations in regional homogeneity of resting-state brain activity in mesial temporal lobe epilepsy. Epilepsia 54: 658-666, 2013.

6. Yang T, Fang Z, Ren J, Xiao F, Li Q, Liu L, Lei D, Gong Q and Zhou D: Altered spontaneous activity in treatment-naive childhood absence epilepsy revealed by regional homogeneity. J Neurol Sci 340: 58-62, 2014.

7. Iwabuchi SJ and Palaniyappan L: Abnormalities in the effective connectivity of visuothalamic circuitry in schizophrenia. Psychol Med 47: 1300-1310, 2017.

8. Yuan X, Han Y, Wei Y, Xia M, Sheng C, Jia J and He Y: Regional homogeneity changes in amnestic mild cognitive impairment patients. Neurosci Lett 629: 1-8, 2016.

9. Ji GJ, Zhang Z, Xu Q, Zang YF, Liao W and Lu G: Generalized tonic-clonic seizures: Aberrant interhemispheric functional and anatomical connectivity. Radiology 271: 839-847, 2014.

10. Zhang Y, Gao Y, Zhou M, Wu J, Zee C and Wang D: A diffusional kurtosis imaging study of idiopathic generalized epilepsy with unilateral interictal epileptiform discharges in children. J Neuroradiol 43: 339-345, 2016.

11. Jensen JH, Helpern JA, Ramani A, Lu H and Kaczynski K: Diffusional kurtosis imaging: The quantification of non-gaussian water diffusion by means of magnetic resonance imaging. Magn Reson Med 53: 1432-1440, 2005.

12. Smith SM, Johansen-Berg H, Jenkinson M, Rueckert D, Nichols TE, Miller KL, Robson MD, Jones DK, Klein JC, Bartsch AJ and Behrens TE: Acquisition and voxelwise analysis of multi-subject diffusion data with tract-based spatial statistics. Nat Protoc 2: 499-503, 2007.

13. Guo YL, Li SJ, Zhang ZP, Shen ZW, Zhang GS, Yan G, Wang YT, Rao HB, Zheng WB and Wu RH: Parameters of diffusional kurtosis imaging for the diagnosis of acute cerebral infarction in different brain regions. Exp Ther Med 12: 933-938, 2016.

14. Kamiya K, Kamagata K, Miyajima M, Nakajima M, Hori M, Tsuruta K, Mori H, Kunimatsu A, Arai H, Aoki S and Ohtomo K: Diffusional kurtosis imaging in idiopathic normal pressure hydrocephalus: Correlation with severity of cognitive impairment. Magn Reson Med Sci 15: 316-323, 2016.

15. Lee CY, Tabesh A, Spampinato MV, Helpern JA, Jensen JH and Bonilha L: Diffusional kurtosis imaging reveals a distinctive pattern of microstructural alternations in idiopathic generalized epilepsy. Acta Neurol Scand 130: 148-155, 2014.

16. Zhang Z, Liao W, Chen H, Mantini D, Ding JR, Xu Q, Wang Z, Yuan C, Chen G, Jiao Q and Lu G: Altered functional-structural coupling of large-scale brain networks in idiopathic generalized epilepsy. Brain 134: 2912-2928, 2011.

17. Engel J Jr and International League Against Epilepsy (ILAE): A proposed diagnostic scheme for people with epileptic seizures and with epilepsy: Report of the ILAE task force on classification and terminology. Epilepsia 42: 796-803, 2001.

18. Chau W and McIntosh AR: The Talairach coordinate of a point in the MNI space: How to interpret it. NeuroImage 25: 408-416, 2005.

19. Li S, Tian J, Bauer A, Huang R, Wen H, Li M, Wang T, Xia L and Jiang G: Reduced integrity of right lateralized white matter in patients with primary insomnia: A diffusion-tensor imaging study. Radiology 280: 520-528, 2016.

20. Rohlfing T: Incorrect ICBM-DTI-81 atlas orientation and white matter labels. Front Neuroscience 7: 4, 2013.

21. Liao W, Zhang Z, Mantini D, Xu Q, Wang Z, Chen G, Jiao Q, Zang YF and Lu G: Relationship between large-scale functional and structural covariance networks in idiopathic generalized epilepsy. Brain Connect 3: 240-254, 2013.

22. Zhu L, Li Y, Wang Y, Li R, Zhang Z, Lu G and Chen H: Aberrant long-range functional connectivity density in generalized tonic-clonic seizures. Medicine (Baltimore) 95: e3893, 2016.

23. Zhang CH, Sha Z, Mundahl J, Liu S, Lu Y, Henry TR and He B: Thalamocortical relationship in epileptic patients with generalized spike and wave discharges-a multimodal neuroimaging study. NeuroImage Clin 9: 117-127, 2015. 
24. Zhong Y, Lu G, Zhang Z, Jiao Q, Li K and Liu Y: Altered regional synchronization in epileptic patients with generalized tonic-clonic seizures. Epilepsy Res 97: 83-91, 2011.

25. Paz JT, Davidson TJ, Frechette ES, Delord B, Parada I, Peng K, Deisseroth K and Huguenard JR: Closed-loop optogenetic control of thalamus as a tool for interrupting seizures after cortical injury. Nat Neurosci 16: 64-70, 2013.

26. Huang W, Lu G, Zhang Z, Zhong Y, Wang Z, Yuan C, Jiao Q, Qian Z, Tan Q, Chen G, et al: Gray-matter volume reduction in the thalamus and frontal lobe in epileptic patients with generalized tonic-clonic seizures. J Neuroradiol 38: 298-303, 2011.

27. Luo C,LiQ,Xia Y,Lei X, Xue K, YaoZ,Lai Y,Martínez-MontesE, Liao W, Zhou D, et al: Resting state basal ganglia network in idiopathic generalized epilepsy. Hum Brain Mapp 33: 1279-1294, 2012 .

28. Ciumas C and Savic I: Structural changes in patients with primary generalized tonic and clonic seizures. Neurology 67: 683-686, 2006.

29. Ciumas C, Wahlin TB, Espino C and Savic I: The dopamine system in idiopathic generalized epilepsies: Identification of syndrome-related changes. NeuroImage 51: 606-615, 2010.

30. Akman O, Gulcebi MI, Carcak N, Ketenci Ozatman S, Eryigit T, Moshé SL, Galanopoulou AS and Onat FY: The role of the substantia nigra pars reticulata in kindling resistance in rats with genetic absence epilepsy. Epilepsia 56: 1793-1802, 2015.

31. Robinson S, Basso G, Soldati N, Sailer U, Jovicich J, Bruzzone L, Kryspin-Exner I, Bauer $\mathrm{H}$ and Moser E: A resting state network in the motor control circuit of the basal ganglia. BMC Neurosci 10 137, 2009.

32. Zhang D, Snyder AZ, Fox MD, Sansbury MW, Shimony JS and Raichle ME: Intrinsic functional relations between human cerebral cortex and thalamus. J Neurophysiol 100: 1740-1748, 2008.

33. Rektor I, Kuba R, Brazdil M and Chrastina J: Do the basal ganglia inhibit seizure activity in temporal lobe epilepsy? Epilepsy Behav 25: 56-59, 2012.

34. Mohan A, Roberto AJ, Lorenzo A, Jones K, Carney MJ, Liogier-Weyback L, Hwang S and Lapidus KA: The significance of the default mode network (DMN) in neurological and neuropsychiatric disorders: A review. Yale J Biol Med 89: 49-57, 2016.

35. Menon V: Large-scale brain networks and psychopathology: A unifying triple network model. Trends Cogn Sci 15: 483-506, 2011

36. Wei H, An J, Shen H, Zeng LL, Qiu S and Hu D: Altered effective connectivity among core neurocognitive networks in idiopathic generalized epilepsy: An fMRI evidence. Front Hum Neurosci 10: 447, 2016.

37. Laureys S, Owen A and Schiff N: Coma science: Clinical and ethical implications. Preface. Prog Brain Res 177: xiii-xiv, 2009.
38. Yoo NJ, Kim HR, Kim YR, An CH and Lee SH: Somatic mutations of the KEAP1 gene in common solid cancers. Histopathology 60: 943-952, 2012.

39. Anastasopoulou S, Kurth F, Luders E and Savic I: Generalized epilepsy syndromes and callosal thickness: Differential effects between patients with juvenile myoclonic epilepsy and those with generalized tonic-clonic seizures alone. Epilepsy Res 129: 74-78, 2017.

40. Matsuo A, Ono T, Baba $\mathrm{H}$ and Ono K: Callosal role in generation of epileptiform discharges: Quantitative analysis of EEGs recorded in patients undergoing corpus callosotomy. Clin Neurophysiol 114: 2165-2171, 2003.

41. Jenssen S, Sperling MR, Tracy JI, Nei M, Joyce L, David G and O'Connor M: Corpus callosotomy in refractory idiopathic generalized epilepsy. Seizure 15: 621-629, 2006.

42. Lee CY, Bennett KM and Debbins JP: Sensitivities of statistical distribution model and diffusion kurtosis model in varying microstructural environments: A monte carlo study. J Magn Reson 230: 19-26, 2013.

43. Kamagata K, Tomiyama H, Hatano T, Motoi Y, Abe O, Shimoji K, Kamiya K, Suzuki M, Hori M, Yoshida M, et al: A preliminary diffusional kurtosis imaging study of parkinson disease: Comparison with conventional diffusion tensor imaging. Neuroradiology 56: 251-258, 2014.

44. Gungor-Tuncer O, Baykan B, Altindag E, Bebek N, Gurses C and Gokyigit A: Prevalence and characteristics of visual aura in idiopathic generalized epilepsy. Epilepsy Behav 25: 573-576, 2012.

45. Sasson E, Doniger GM, Pasternak O, Tarrasch R and Assaf Y: White matter correlates of cognitive domains in normal aging with diffusion tensor imaging. Front Neurosci 7: 32, 2013.

46. Du H, Zhang Y, Xie B, Wu N, Wu G, Wang J, Jiang T and Feng H: Regional atrophy of the basal ganglia and thalamus in idiopathic generalized epilepsy. J Magn Reson Imaging 33: 817-821, 2011.

47. Walser G, Unterberger I, Dobesberger J, Embacher N, Falkenstetter T, Larch J, Kuchukhidze G, Gotwald T, Ortler M, Bauer G and Trinka E: Asymmetric seizure termination in primary and secondary generalized tonic-clonic seizures. Epilepsia 50: 2035-2039, 2009.

This work is licensed under a Creative Commons Attribution-NonCommercial-NoDerivatives 4.0 International (CC BY-NC-ND 4.0) License. 